\title{
Laboreal
}

Volume $6 \mathbf{N}^{\circ} 1$ | 2010

Varia

\section{Produzir a saúde, produzir a segurança : desenvolver uma cultura colectiva de segurança em radioterapia}

Producir salud, producir seguridad: desarrollar una cultura colectiva de seguridad en la radioterapia

Produire la santé, produire la sécurité : développer une culture collective de sécurité en radiothérapie

Producing health, producing safety: developing a collective safety culture in radiotherapy

\section{Adelaide Nascimento}

\section{(2) OpenEdition}

\section{Journals}

\section{Edição electrónica}

URL: http://journals.openedition.org/laboreal/9180

DOI: 10.4000/laboreal.9180

ISSN: 1646-5237

\section{Editora}

Universidade do Porto

\section{Refêrencia eletrónica}

Adelaide Nascimento, «Produzir a saúde, produzir a segurança : desenvolver uma cultura colectiva de segurança em radioterapia », Laboreal [Online], Volume $6 N^{0} 1 \mid$ 2010, posto online no dia 01 julho 2010, consultado o 24 setembro 2020. URL : http://journals.openedition.org/laboreal/9180 ; DOI : https:// doi.org/10.4000/laboreal.9180

Este documento foi criado de forma automática no dia 24 setembro 2020.

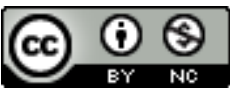

Laboreal está licenciado com uma Licença Creative Commons - Atribuição-NãoComercial 4.0 Internacional. 


\section{Produzir a saúde, produzir a segurança : desenvolver uma cultura colectiva de segurança em radioterapia}

Producir salud, producir seguridad : desarrollar una cultura colectiva de seguridad en la radioterapia

Produire la santé, produire la sécurité : développer une culture collective de sécurité en radiothérapie

Producing health, producing safety: developing a collective safety culture in radiotherapy

Adelaide Nascimento

\section{REFERÊNCIA}

Nascimento, A. (2009). Produire la santé, produire la sécurité. Développer une culture collective de sécurité en radiothérapie. Thèse de Doctorat en Ergonomie, Conservatoire National des Arts et Métiers, Paris.

\section{NOTA DO EDITOR}

Manuscrito recebido em: Abril/2010

Aceite após peritagem: Junho/2010 


\section{NOTA DO AUTOR}

6 operadores vindos de 3 hospitais diferentes participaram desta fase do estudo. Além das entrevistas, nenhum estudo aprofundado foi realizado nos hospitais dos quais eles fazem parte.

\section{Problemática: melhorar a cultura de segurança em radioterapia}

1 A segurança dos pacientes, considerada como o fato de "estar protegido contra acidentes hospitalares” (Kohn, Corrigan, \& Donaldson, 2000, pp. 4, tradução livre) tornou-se um tema bastante midiatizado nesses últimos anos, nomeadamente depois da publicação do relatório americano "To err is human: building a safer health care system" em 1999 (Kohn, Corrigan,

2 \& Donaldson, 2000). Esta publicação, assim como os impactos sociais e midiáticos que a acompanharam, representa para a segurança dos pacientes o mesmo que o acidente de Three misles Island (1979) representa para a indústria nuclear. Com efeito, o relatório americano é considerado como o ponto de partida para a tomada de consciência coletiva sobre a necessidade de melhorar a segurança dos pacientes (Leape, 2004). Nele é denunciada a falta de "cultura de segurança" no meio médico, principalmente no que concerne os comportamentos dos profissionais de saúde.

3 A falta de "cultura de segurança" foi um dos termos encontrados pelas autoridades nucleares francesas para explicar o maior acidente de radioterapia já visto no mundo: acidente ocorrido em 2006 em Epinal (França), onde 23 pacientes morreram e mais de 4 mil ficaram com sequelas devido a doses de radiação inadequadas (Peiffert, Simon, \& Eschwege, 2007). As inspeções do acidente de 2006 conduziram à descoberta de mais dois outros acidentes, ocorridos no mesmo hospital entre 2001 e 2006. As falhas técnicas e organizacionais que causaram "os acidentes" de Epinal datavam de 1989 (ASNIGAS, 2007).

O presente trabalho de tese trata da segurança dos pacientes e da cultura de segurança no campo da radioterapia, especialidade médica que utiliza radiações ionizantes no tratamento do câncer. Apesar dos altos índices de cura obtidos atualmente em radioterapia (em torno de 40\%) (ASN, 2007), alguns riscos de acidente, se não controlados, podem conduzir a consequências graves para a saúde dos pacientes, como nos mostra os acidentes ocorrido na França e em outras partes do mundo (Cosset \& Gourmelon, 2002). A demanda da pesquisa emana do Serviço de Estudos sobre os Fatores Humanos (SEFH) do Institut de Radioprotection et de Sûreté Nucléaire (IRSN). Trata-se igualmente de uma demanda social relativa a melhoria da cultura de segurança em radioterapia.

\section{Quadro teórico: Cultura de segurança e trabalho coletivo}

5 Na sua definição original, a cultura de segurança seria uma característica dos indivíduos mas também das organizações: "conjunto de características e atitudes dos indivíduos e das organizações que fazem com que as questões relativas à segurança 
sejam tratadas com a prioridade e atenção que elas merecem, em razão da importância que elas têm" (INSAG-4, 1991, pp. 1, tradução livre). Utilizando a literatura francesa e internacional sobre a confiabilidade humana e organizacional (Amalberti, 1996; Hollnagel, 2008; Rasmussen, 1997; Reason, 1993), nós consideramos que a cultura de segurança é uma característica dos indivíduos, das organizações, de um grupo de profissionais, mas também que ela é uma característica transversa às diferentes profissões. Esta visão nos conduz a levar em consideração a dimensão coletiva da atividade de trabalho (Barthe \& Quéinnec, 1999; De la Garza \& Weill-Fassina, 2000; Leplat, 1993). O coletivo, assim como o Homem isolado, aparece na literatura tanto como uma fonte de falha como um recurso suplementar para a segurança (Marc \& Amalberti, 2002).

Em radioterapia, a preparação e a administração dos tratamentos compreendem 11 etapas sucessivas e resultam de um trabalho de coordenação e cooperação importante entre 4 tipos de profissionais diferentes: radioterapêutas, físicos médicos, dosimetristas e técnicos em radioterapia. Em contextos deste tipo, onde os profissionais que compartilham o mesmo objetivo global são oriundos de formações diferentes e possuem práticas profissionais específicas, o desenvolvimento do coletivo seguro tornase fundamental. Ele visa por um lado prevenir o aparecimento de situações perigosas a serem geradas pelos colegas, e por outro lado aumentar a recuperação dessas situações, caso elas ocorram (Sasou \& Reason, 1999). Nós nomeamos de "cultura coletiva de segurança" a consciência relativa aos riscos e perigos dos tratamentos compartilhada entre todos os grupos de profissionais. Ela é baseada na importância da atividade de todos na atividade de cada um.

O objetivo conceitual desta tese foi compreender como os profissionais da radioterapia gerenciam, individualmente e coletivamente, as obrigações e os recursos disponíveis afim de responder aos objetivos de produção da saúde e produção da segurança dos pacientes sabendo-se que em alguns casos esses dois objetivos podem entrar em contradição. A partir desta compreensão e enriquecimento dos modelos existentes na literatura, o objetivo pragmático desta tese foi fornecer vias de melhoria da segurança dos pacientes através do desenvolvimento do que nomeamos de "cultura coletiva de segurança", cujo objetivo maior é o desenvolvimento do coletivo seguro.

\section{Métodos complementares, campos de pesquisa distintos}

8 A pesquisa foi realizada em sua grande parte em dois hospitais da região parisiense, diferentes em termos de financiamento (um publico (A), um privado (B)) e de acesso à novas tecnologias (um centro de ponta (A) e outro convencional (B)). Depois de uma fase de imersão (180h de observações abertas), três estudos empíricos foram realizados.

9 Levando-se em conta as diferentes profissões envolvidas, nosso objetivo inicial foi verificar e identificar a diversidade de práticas através do julgamento de situações de desvio às regras. 14 operadores do hospital A (radioterapeutas, físicos médicos, dosimetristas e técnicos em radioterapia) analisaram 9 situações reais de desvios às regras obtidas durante a fase de observação aberta; em seguida, nós nos interessamos a melhor compreender a procedência e a recuperação das situações de desvio às regras. Neste sentido, o posto de trabalho dos técnicos em radioterapia foi considerado como 
um ambiente propício por se situar no fim da "cadeia do tratamento" (posto de administração do tratamento). A atividade dos técnicos em radioterapia - e sua ligação com o coletivo - foi analisada através de observações sistemáticas in situ realizadas nos dois hospitais. $O$ interesse era de ter acesso ao trabalho real e as regulações individuais e coletivas realizadas por esses profissionais no cotidiano; por fim, os resultados do segundo estudo empírico nos conduziram à analisar a atividade dos físicos médicos. 0 objetivo era identificar as estratégias de prevenção de riscos colocadas em prática pelos físicos médicos em caso de tratamentos difíceis de serem executados pelos técnicos de radioterapia. 14 físicos médicos, de 5 hospitais diferentes (A, B, C, D,

E) puderam, graças ao método de allo-confrontação individual, comentar dois planos de tratamento reais realizados por colegas. Foi possível assim identificar um conjunto de estratégias visando não somente a prevenção, mas o controle das situações arriscadas que não podem ser prevenidas.

11 Com o objetivo de obter uma validação dos resultados pelos profissionais da radioterapia, uma restituição coletiva foi organizada em um dos hospitais envolvidos na pesquisa (hospital A). Para acompanhar a pesquisa de maneira global, um comitê de pilotagem, contando com a participação de profissionais da ergonomia e da radioterapia, foi constituído e se reuniu 2 vezes por ano durante os 3 anos de tese.

\section{Resultados e Discussão}

\subsection{A gestão dos conflitos entre qualidade e segurança}

Os resultados desta tese evidenciam que a confiabilidade dos tratamentos em radioterapia compreende não somente a segurança dos pacientes, mas também a qualidade dos cuidados. A gestão dos conflitos entre qualidade e segurança é fruto da segurança em ação, ou seja da "maneira encontrada pelos operadores para agir em segurança face as perturbações e para gerenciar suas próprias ações, que nem sempre são ideais com relação as regras" (De Terssac \& Gaillard, 2009, pp. 14, tradução livre).

Durante a fase de observação sistemática da atividade dos técnicos em radioterapia, nós identificamos situações de desvio às regras (ausência de validação de um dossiê pelo médico responsável, por exemplo) conduzindo à um conflito entre a qualidade dos cuidados (saúde) e a segurança dos pacientes. No caso da ausência de validação medical, se o tratamento é realizado, o objetivo de qualidade é atingido pois o paciente recebeu o seu tratamento do dia. Porém, não se pode garantir que este tratamento corresponda ao desejo do médico, já que este não o validou. Se o tratamento é anulado, não há risco de erro. $O$ objetivo de segurança é atingido, mas não o de qualidade. $O$ que devem fazer os técnicos? Para a tomada de decisão, o conhecimento do trabalho dos colegas apareceu como um dado fundamental: os técnicos levam em consideração os hábitos e regras informais dos colegas de serviço (o médico não valida o dossiê pois ele confia no físico médico que o preparou, por exemplo) para tomar a decisão que melhor responde ao equilíbrio entre saúde e segurança dos pacientes. Eles consideram igualmente o estado do paciente. Notamos assim a presença da segurança em ação ou gerenciada, ou seja, que não se baseia em regras formais, escritas, mas em regras implícitas, construídas e possibilitadas pela experiência no serviço e pelo conhecimento do trabalho dos colegas (Amalberti, 2007; Daniellou, 2008; Nascimento, 2009; Nascimento \& Falzon, 2009). 
14 A partir das entrevistas de allo-confrontação com os físicos médicos, foi colocado em evidência que em caso de conflito entre a qualidade dos cuidados e a segurança dos pacientes, a grande maioria dos operadores entrevistados tendem a privilegiar a qualidade do tratamento (então a saúde dos pacientes) em detrimento da segurança. Com efeito, eles preferem escolher um plano de tratamento que aumente as chances de cura do câncer, mesmo se este é muito difícil a ser realizado pelos técnicos em radioterapia em vez de um tratamento de fácil execução, mas de pouca eficácia clínica. Porém, os físicos fazem esta escolha porque eles dispõem de estratégias destinadas a prevenir os riscos de execução dos tratamentos, com por exemplo, a consideração das dificuldades eventuais encontradas pelos técnicos em radioterapia durante a sessão. Assim, a qualidade "regrada", vinda dos referenciais terapêuticos mais modernos, é corroborada pela segurança "gerenciada", isto é, pelas estratégias de segurança informais desenvolvidas e colocadas em prática pelos físicos médicos.

\subsection{A existência de subculturas de segurança segundo as profissões e os hospitais}

A partir da análise de situações reais de desvio às regras, foi colocado em evidência a existência de culturas de segurança distintas segundo a atividade dos operadores. Os profissionais diferem quanto à tolerância aos desvios ou quanto aos desvios que eles toleram, sendo os médicos os mais tolerantes e os técnicos de radioterapia os menos. Este mecanismo cultural se traduz como uma ausência de acordo no âmbito do coletivo no que se refere a significação dada as coisas e aos eventos (Grote \& Künzler, 2000). Isto pode conduzir à situações arriscadas quando os diferentes pontos de vistas, arraigados em uma dada profissão, não são postos em comum, nem discutidos coletivamente (Falzon, 2005).

16 Quanto aos hospitais, foi identificado que as decisões face ao risco dependem dos meios humanos e tecnológicos disponíveis. Como os hospitais estudados tinham modos de funcionamento e tecnologias diferentes, as arbitragens entre qualidade e segurança também diferem de um hospital a outro, o que subentende culturas de segurança próprias.

\section{Da cultura de segurança à cultura coletiva de segurança: a importância do trabalho de todos no trabalho de cada um}

17 Os resultados desta pesquisa mostram uma visão da segurança total que engloba qualidade e segurança, gerenciadas ou regradas. Por um lado, nós colocamos em evidência o coletivo como um fator de confiabilidade (antecipação das dificuldades dos colegas pelos físicos médicos, recuperação das situações de desvio às regras pelos técnicos, etc.). É a manifestação do que chamamos de cultura coletiva de segurança. Por outro lado, foi evidenciado o papel do coletivo como um fragilizador da segurança (diversidade de práticas não compartilhadas, acordos de confiança entre ofícios com impacto na atividade de um dado profissional, etc.). Isto abre perspectivas quanto ao desenvolvimento do coletivo seguro. 
neira geral, e afim de poder assegurar a segurança total em radioterapia, ou seja a produção da qualidade (saúde) em segurança, é primordial fornecer às organizações recursos humanos e materiais importantes, mas também um lugar privilegiado para o desenvolvimento do coletivo e da organização prescrita. Com efeito, nossa pesquisa evidencia a existência de poucas regras formais em radioterapia, concordando assim com resultados de outros estudos (Thellier, Regnier \& Derreumaux, 2008). No entanto, foi verificada a existência de regras securitárias informais, implícitas, construídas no seio dos coletivos de trabalho. Algumas dessas regras não escritas podem vir alimentar a prescrição, desde que elas sejam discutidas coletivamente. Construir e desenvolver uma cultura coletiva de segurança supõe favorecer a construção de espaços de deliberação permitindo aos atores implicados de fazer valer suas próprias dificuldades e exigências, de descobrir estas dos seus colegas e de decidir conjuntamente sobre as regras e a autonomia necessárias; trata-se então de conceber um ambiente de trabalho capacitante (Pavageau, Nascimento, \& Falzon, 2007), tanto para os indivíduos quanto para o coletivo e a organização.

\section{BIBLIOGRAFIA}

Amalberti, R. (1996). La conduite de systèmes à risques. Paris: PUF Amalberti, R. (2007). Ultrasécurité. Une épée de Damoclès pour les hautes technologies. Sciences à risques. Les dossiers de la recherche $n^{\circ} 26,74-81$.

ASNIGAS. (2007). Résumé du rapport ASN n²006 ENSTR 019 - IGAS, n RM 2007-015P sur l'accident de radiothérapie d'Epinal: Inspection Générale des Affaires Sociales.

ASN. (2007). Guide ASN/DEU/03 relatif aux modalités de déclaration et à la codification des critères relatifs aux événements significatifs dans le domaine de la radioprotection hors installations nucléaires de base et transport de matières radioactives - version du 15/06/2007.

Barthe, B., \& Quéinnec, Y. (1999). Terminologie et perspectives d'analyse du travail collectif en Ergonomie. L'Année psychologique, 99, 663-686.

Cosset, J.-M., \& Gourmelon. (2002). Accidents en radiothérapie: un historique. Cancer/Radiother 6(Suppl. 1), 166-170.

Daniellou, F. (2008). Développement des TMS: désordre dans les organisations et fictions mangériales. Paper presented at the 2ème Congrès francophone sur les toubles musculosquelettiques: de la recherche à l'action.

De la Garza, C., \& Weill-Fassina, A. (2000). Régulations horizontales et verticales du risque. In T. H. Benchekroun \& A. Weill-Fassina (Eds.), Le travail collectif. Perspectives actuelles en ergonomie (pp. 217-224). Toulouse: Octarès Editions.

De Terssac, G., \& Gaillard, I. (2009). Règle et sécurité: partir des pratiques pour définir les règles? In G. de Terssac, I. Boissières \& I. Gaillard (Eds.), La sécurité en action (pp. 13-34). Toulouse: Octarès. 
Falzon, P. (2005). Ergonomics, knowledge development and the design of enabling environments. Humanizing Work and Work Environment Conference. December 10-12, Guwahati, India.

Grote, G., \& Künzler, C. (2000). Diagnosis of safety culture in safety management audits. Safety Science, 34(1-3), 131-150.

Hollnagel, E. (2008). Risk + barriers = safety? Safety Science, 46(2), 221-229.

INSAG-4. (1991). Safety culture: International Nuclear Safety Advisory Group - AIEA.

Kohn, L. T., Corrigan, J. M., \& Donaldson, M. S. (2000). To err is human: building a safer health care system. Washington, D.C.: National Academy Press.

Leape, L. L. (2004). Making health care safe: are we up to it? Journal of Pediatric Surgery, 39(3), 258-266.

Leplat, J. (1993). Ergonomie et activités collectives. In F. Six \& X. Vaxevanoglou (Eds.), Les aspects collectifs du travail (pp. 7-27). Toulouse: Octarès.

Marc, J., \& Amalberti, R. (2002). Contribution individuelle à la sécurité du collectif: l'exemple de la régulation du samu. Le Travail Humain, 65(3), 217-242.

Nascimento, A. (2009). Produire la santé, produire la sécurité. Développer une culture collective de sécurité en radiothérapie. Unpublished Thèse de Doctorat en Ergonomie, Conservatoire National des Arts et Métiers, Paris.

Nascimento, A., \& Falzon, P. (2009). Produire la santé, produire la sécurité: récupérations et compromis dans le risque des manipulatrices en radiothérapie. Activités 6(2), 3-23. http:// www.activites. org/v26n22/v26n22.pdf.

Pavageau, P., Nascimento, A., \& Falzon, P. (2007). Les risques d'exclusion dans un contexte de transformation organisationnelle. PISTES, 9(2), http://www.pistes.uqam.ca/v9n2/articles/ v9n2a6. htm.

Peiffert, D., Simon, J. M., \& Eschwege, F. (2007). L'accident d'Épinal: passé, présent, avenir. Cancer/Radiothérapie, 11(6-7), 309-312.

Rasmussen, J. (1997). Risk management in a dynamic society: a modelling problem. Safety Science, 27(2-3), 183-213.

Reason, J. (1993). L'erreur humaine. Paris PUF.

Sasou, K., \& Reason, J. (1999). Team errors: definition and taxonomy.

Reliability Engineering \& System Safety, 65(1), 1-9.

Thellier, S., Regnier, P., \& Derreumaux, S. (2008). Améliorer la sécurité des traitements en radiothérapie en développant une culture de sûreté (No. 2008-2): rapport de l'Institut de Radioprotection et Sûreté Nucléaire (IRSN).

\section{AUTOR}

\section{ADELAIDE NASCIMENTO}

CNAM - Centre de Recherche sur le Travail et le Développement (CRTD) - Equipe Ergonomie 41, rue Gay-Lussac 75005 Paris adelaide.nascimento@cnam.fr 